\title{
E/e' ratio Predicts the Atrial Pacing-induced Left atrial Pressure Response in Pa- tients with Preserved Ejection Fraction
}

\author{
Seung-Young Roh ${ }^{1,+}$, Kwang-No Lee ${ }^{2,+}$, Yong-Soo Baek ${ }^{3}$, Mohammad Iqbal ${ }^{4}$, Anupam Jena ${ }^{5}$, Saruul Tseveendee ${ }^{6}$, \\ Jaemin Shim ${ }^{1}$, Jong-Il Choi ${ }^{1}$ and Young-Hoon Kim ${ }^{1, *}$
}

1 Division of Cardiology, Department of Internal Medicine, Korea University College of Medicine and Korea University Medical Center, Seoul, Republic of Korea; rsy008@gmail.com (S-Y.R.); jaemins@korea.ac.kr (J.S.) jongilchoi@korea.ac.kr (J-I.C.)

2 Division of Cardiology, Department of Internal Medicine, Ajou University Hospital, Suwon, Republic of Korea; knlee81@gmail.com

3 Division of Cardiology, Department of Internal Medicine, Inha University hospital, Incheon, Republic of Korea; existsoo@hanmail.net

4 Division of Cardiology, Department of Internal Medicine, Dr. Hasan Sadikin Central General Hospital, University Padjadjaran, Kota Bandung, Indonesia; mohammadiqbal178@gmail.com

5 Division of Cardiology, Department of Internal Medicine, Kalinga institute of Medical Sciences, Bhubaneswar, India; dranupamjena@gmail.com

6 Division of Cardiology, Department of Internal Medicine, Third State Hospital, Ulaanbaatar, Mongolia; tssrlmd@gmail.com

* Correspondence: yhkmd@korea.ac.kr

+ These authors contributed equally to the study.

Abstract: Introduction: Left atrial hypertension is one of the pathophysiologies of heart failure with preserved ejection fraction. We hypothesized that left atrial pressure response (LAPR) to incremental pacing is higher in patients with atrial fibrillation (AF) and can predict left ventricular diastolic dysfunction. Methods: Patients requiring left atrial access as a part of a therapeutic procedure for $\mathrm{AF}$ ( $\mathrm{n}=204$, AF group) or supraventricular tachycardia ( $\mathrm{n}=34$, control group) were analyzed (male $\mathrm{n}=183,54 \pm 12$ years old). LAPR was measured during incremental pacing. Results: Baseline left atrial pressure and LAPR at all pacing rates were not different between the AF and control groups. They were higher in patients with a high E/e' $(\geq 8)$ than in those with a low E/e' $(<$ 8). LAPR at a pacing interval of $400 \mathrm{~ms}$ and $\mathrm{E} / \mathrm{e}^{\prime}$ were positively correlated $(\mathrm{r}=0.373, \mathrm{p}<0.001)$. Body mass index and a high E/e' were independent predictors of pacing-induced left atrial hypertension. Conclusions: The LAPR to incremental pacing was constant regardless of AF. The non-invasive echocardiographic marker E/e' reflected pacing-induced left atrial hypertension.

Keywords: diastolic dysfunction; heart failure; atrial fibrillation; atrial hypertension; left atrial pressure

\section{Introduction}

Heart failure (HF) is one of the leading causes of hospitalization and cardiovascular mortality regardless of the left ventricular (LV) ejection fraction (EF). Several studies have demonstrated similar mortality rates between individuals having HF with preserved EF (HFpEF) and those having HF with reduced EF. [1,2] The main hemodynamic pathophysiology of HFpEF is the elevation of LV filling pressure (LVFP). In patients with advanced HFpEF, LVFP is elevated at rest. However, in the early stage, increased LVFP is observed only during strenuous physical activity. [3] High LVFP during exercise in HFpEF is associated with symptoms such as dyspnea and aerobic capacity reduction. If HFpEF progresses over time, left atrial (LA) remodeling and dysfunction develop. 
Thus, LA remodeling reflects the cumulative effects of elevated LVFP. Elevated LA pressure is related to LA remodeling in the general population regardless of atrial fibrillation (AF). It provides diagnostic and prognostic information about LV diastolic dysfunction and the chronicity of the disease. A recent study showed that elevated LA pressure is associated with extended electro-anatomical remodeling of the LA and poor clinical outcomes after AF ablation. [4] In addition, it is known to trigger AF by causing ectopic beats emanating from the pulmonary veins (PVs). [5] However, invasively measured LA pressure is insufficient to identify the stage of HFpEF because it is usually not increased in the early stage because of LA adaptation. In addition, it is not a fixed parameter because it is sensitive to the body volume and heart rate. [6]

Therefore, we hypothesized that the LA pressure response (LAPR) to incremental pacing reflects LV diastolic dysfunction. The objective of this study was to reveal the clinical implications and non-invasive predictors of LAPR.

\section{Methods}

\subsection{Study protocol}

We screened a population who needed LA access as a part of a therapeutic procedure for AF or supraventricular tachycardia (Figure 1). From July 2015 to November 2016, 264 patients with AF were enrolled. The control group consisted of 35 patients with re-entry tachycardia via left side accessory pathway or left origin atrial tachycardia. Patients with (1) previous cardiac surgery or procedure history $(n=0)$; (2) LV systolic dysfunction $(\mathrm{LVEF}<50 \%)$ or structural heart disease including ischemic lesion $(\mathrm{n}=15),(3)$ moderate to severe mitral valve disease $(n=0),(4)$ recurrent triggers, that induced sustained arrhythmias interrupting the maintain sinus rhythm (SR) $(n=1)$, and (5) AF induction during right atrial pacing ( $n=14$ in AF group and $n=1$ in control group) were excluded via a screening test. A total of 204 patients in the AF group and 34 patients in the control group were finally analyzed (male $77.1 \%, 54.0 \pm 12.4$ years old). In addition, the cohort was divided to two groups based on criteria of $\mathrm{E} / \mathrm{e}^{\prime}=8$ (median value), which is an echocardiographic LV diastolic dysfunction marker. We compared 144 patients with low $\mathrm{E} / \mathrm{e}^{\prime}$ and 124 patients with high E/e'. All patients provided written informed consent for inclusion in the cohort. The research protocol complied with the principles of the Declaration of Helsinki and was approved by the Institutional Review Board of the Korea University Anam Hospital (2016AN0152). 


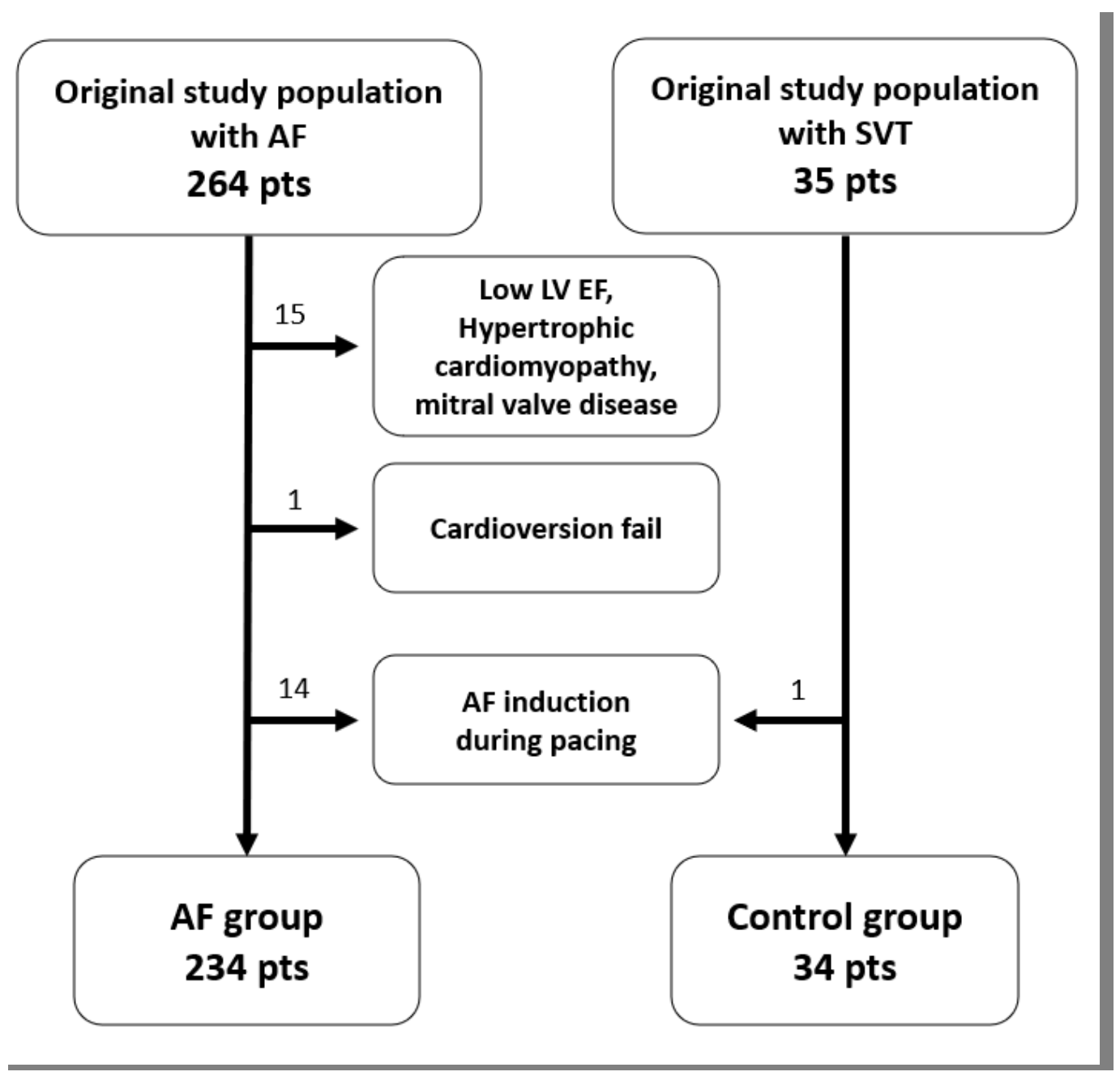

Figure 1. The diagram demonstrating patient enrollment and disposition during the study; AF, atrial fibrillation; SVT, supraventricular tachycardia; LV, left ventricular; EF, ejection fraction.

\subsection{Echocardiography}

All candidates underwent transthoracic echocardiography within a month prior to the procedure. Cardiac chamber size, LV wall thickness, blood flow velocity, and tissue Doppler images of the mitral annular septal region were assessed. The E wave represents the ratio of peak velocity of blood flow from left ventricular relaxation in early diastole. $\mathrm{e}^{\prime}$ is a measure of peak mitral annular velocity during early filling. Of the 268 enrolled patients, 164 had SR and 104 had AF at the time of transthoracic echocardiography.

\subsection{Measurements of left atrial pressure and incremental pacing}

All patients remained fasting for $8 \mathrm{~h}$ prior to invasive LA pressure measurement. The procedure was performed under sedation using propofol. We did not use general anesthesia. Intracardiac echocardiography and measurement of hemodynamics were performed using a Prucka CardioLab electrophysiology recording system (General Electric Medical system Inc., Milwaukee, WI, USA). A septal puncture was performed to assess the LA. Systemic anticoagulation was initiated with intravenous heparin, maintaining an 
active coagulation time of 300 to $350 \mathrm{~s}$ immediately before septal puncture. A Swartz left 1 long sheath (St. Jude Medical, Inc. Minnetonka, MN, USA) was used for septal puncture. To measure LA pressure, a 6-F pigtail catheter (A \& A Medical Devices Inc. Gyeonggi-do, Korea) was inserted into the LA through the long sheath. Baseline LA pressure was measured during SR at the height of the v wave. If AF was sustained at the initial time of the procedure, SR was restored with an internal cardioversion (Physio-Control Lifepack 12, Physio-Control Corp., Redmond, WA, USA) with 5-20 J of energy and LA pressure was measured 5 min after restoring SR. To increase the heart rate, incremental right atrial pacing was performed. The LAPRs at heart rates of 60, 75, 100, 120 , and 150 beats per minute (bpm) were observed. If the patient's breathing was unstable, LA pressure was measured during inspiration.

\subsection{Statistical analyses}

Statistical analyses were performed using the SPSS 20.0 software package (SPSS Inc., Chicago, IL, USA). Continuous variables are expressed as mean \pm standard deviation. They were compared by Student's t-test, Mann-Whitney U-test, and ANOVAs, followed by post hoc analyses using Bonferroni's method. Categorical variables are reported as counts with percentages and were compared using a chi-square test or Fisher's exact test. The difference in pacing-dependent LA pressure changes was determined by ANOVA. Multivariate analysis was conducted with a logistic regression model reporting odds ratios (ORs) to predict high LAPR (LA pressure $\geq 26 \mathrm{mmHg}$ ). Predictor variables included age, female sex, hypertension, diabetes mellitus, atrial fibrillation, body mass index, LA diameter, LV mass index, LVEF, and high E/e'. Multiple regression analysis was performed using the criterion of $p<0.10$ in the univariate analyses for a variable to enter the model. AF/atrial tachycardia (AT)-free survival was measured by the Kaplan-Meier survival curve analysis, and the difference between both groups was assessed by a log-rank test. A p-value $<0.05$ was considered statistically significant.

\section{Results}

\subsection{Baseline characteristics}

The baseline characteristics of the AF and control groups are presented in Table 1. The AF group was older $(56 \pm 11$ vs. $39 \pm 14$ years, $\mathrm{p}<0.001)$ and included more men $(81$ vs. $59 \%$, $\mathrm{p}=0.007)$. This group had a higher BMI $\left(25 \pm 3 \mathrm{vs.} 23 \pm 3 \mathrm{~kg} / \mathrm{m}^{2}, \mathrm{p}=0.003\right)$ and more hypertension ( 38 vs. $6 \%, \mathrm{p}<0.001)$. In echocardiographic data, the AF group had a larger LV diastolic diameter ( $47 \pm 4$ vs. $45 \pm 4 \mathrm{~mm}, \mathrm{p}=0.026)$, higher LV mass $(161 \pm 30$ vs. $129 \pm 29 \mathrm{~g}$, $\mathrm{p}<0.001)$, larger LA anteroposterior diameter $(41 \pm 6$ vs. $32 \pm 4 \mathrm{~mm}, \mathrm{p}<0.001)$, and higher $\mathrm{E} / \mathrm{e}^{\prime}(8 \pm 3$ vs. $7 \pm 2, \mathrm{p}=0.003)$. The $\mathrm{LVEF}$ was preserved, and there were no significant differences between the two groups.

Table 1. Demographics and clinical characteristics of study population

\begin{tabular}{ccc}
\hline AF group & Control & $p$-value \\
$\mathrm{n}=234$ & $\mathrm{n}=34$ & \\
\hline
\end{tabular}




\begin{tabular}{lccc} 
Age, y (SD) & $56(11)$ & $39(14)$ & $<0.001$ \\
Male (\%) & $189(81)$ & $20(59)$ & 0.007 \\
Body mass index, kg/m² (SD) & $25(3)$ & $23(3)$ & 0.003 \\
Hypertension (\%) & $88(38)$ & $2(6)$ & $<0.001$ \\
Diabetes mellitus (\%) & $21(9)$ & 0 & 0.051 \\
Myocardial infarction (\%) & $4(2)$ & 0 & 0.579 \\
Cerebrovascular accident (\%) & $13(6)$ & 0 & 0.164 \\
History of heart failure (\%) & $5(2)$ & 0 & 0.505 \\
Chronic kidney disease (\%) & $4(2)$ & 0 & 0.579 \\
Thyroid disease (\%) & $9(4)$ & 0 & 0.289 \\
Echocardiographic data & & & \\
LVID in diastole, mm (SD) & $47(4)$ & $45(4)$ & 0.026 \\
LVID in systole, mm (SD) & $29(5)$ & $28(3)$ & 0.532 \\
LV mass, g (SD) & $161(30)$ & $129(29)$ & $<0.001$ \\
LV mass index, g/m² (SD) & $89(16)$ & $75(15)$ & $<0.001$ \\
LV Ejection fraction, \% (SD) & $59(3)$ & $60(2)$ & 0.054 \\
LA AP diameter, mm (SD) & $41(6)$ & $32(4)$ & $<0.001$ \\
E (SD) & $63(15)$ & $64(14)$ & 0.584 \\
e' (SD) & $8(2)$ & $9(3)$ & 0.045 \\
E/e' ratio (SD) & $8(3)$ & $7(2)$ & 0.003 \\
DT of E (SD) & $173(40)$ & $181(35)$ & 0.230 \\
Estimated PASP, mmHg (SD) & $29(6)$ & $27(4)$ & 0.235 \\
\hline
\end{tabular}

$\mathrm{AF}=$ atrial fibrillation; $\mathrm{LVID}=$ left ventricular internal diameter; $\mathrm{LV}=$ left ventricular; $\mathrm{LA}$ $=$ left atrial; $\mathrm{AP}=$ anterorposterior; $\mathrm{DT}=$ deceleration time; $\mathrm{PASP}=$ pulmonary artery systolic pressure.

\subsection{High E/e' group versus low E/e' group}

We additionally analyzed the difference between patients with high E/e' and low E/e' (Table 2). The high $E / e^{\prime}$ group was older ( $58 \pm 11$ vs. $50 \pm 12$ years, $\left.\mathrm{p}<0.001\right)$, included fewer men (67 vs. $88 \%, p<0.001)$, and had a greater number of hypertensive $(45 \%$ vs. $24 \%$, $\mathrm{p}<0.001)$ and diabetic $(13 \%$ vs. $4 \%, \mathrm{p}=0.005)$ patients than the low $\mathrm{E} / \mathrm{e}^{\prime}$ group. The high $\mathrm{E} / \mathrm{e}^{\prime}$ group had more common history of myocardial infarction ( $3 \%$ vs. $\left.0 \%, \mathrm{p}=0.045\right)$. In echocardiographic data, the high E/e' group had a higher LV mass index ( $91 \pm 16$ vs. $85 \pm 16 \mathrm{~g} / \mathrm{m}^{2}, \mathrm{p}=0.001$ ), a larger LA anteroposterior diameter ( $41 \pm 6$ vs. $38 \pm 6 \mathrm{~mm}, \mathrm{p}<0.001$ ), and higher pulmonary artery systolic pressure ( $30 \pm 6 \mathrm{vs.} 28 \pm 6 \mathrm{mmHg}, \mathrm{p}=0.002)$. Baseline LA pressure was not significantly different between the high E/e' group and low E/e' group.

\subsection{The left atrial pressure response by right atrial pacing}

The heart rate changed according to the right atrial pacing (RAP) interval (Figure 2). LA pressure did not increase at $75 \mathrm{bpm}$ and $100 \mathrm{bpm}$, but rose at $120 \mathrm{bpm}$ and $150 \mathrm{bpm}$. A similar pattern was observed in both the AF and control groups. LA pressure was not different at any of the heart rates in both groups. In the High E/e' group and the Low 
E/e' group, the LAPR differed according to RAP (Figure 3). There was a significant difference at all pacing rates. In addition, the difference was more pronounced at $400 \mathrm{~ms}$ than at baseline (11\% vs 16\%). Both LA pressure at baseline and $400 \mathrm{~ms}$ were related to $\mathrm{E} / \mathrm{e}^{\prime}$ and positively correlated ( $\mathrm{r}=0.230$ and $\mathrm{r}=0.373$ ) (Figure 4). Linear regression was performed to find the predictor of high LAPR (LA pressure $\geq 27 \mathrm{mmHg}$ ) (Table 3). BMI, LV mass index, and high E/e' ( $<<0.10$ in the univariate analysis) were included in the multivariate analysis. $\mathrm{BMI}(\mathrm{OR}=1.098$ [1.006-1.197], $\mathrm{p}=0.035)$ and high $\mathrm{E} / \mathrm{e}^{\prime}(\mathrm{OR}=2.054$ [1.235-3.416], $\mathrm{p}=0.006)$ were independent predictors of high LAPR.

(A)

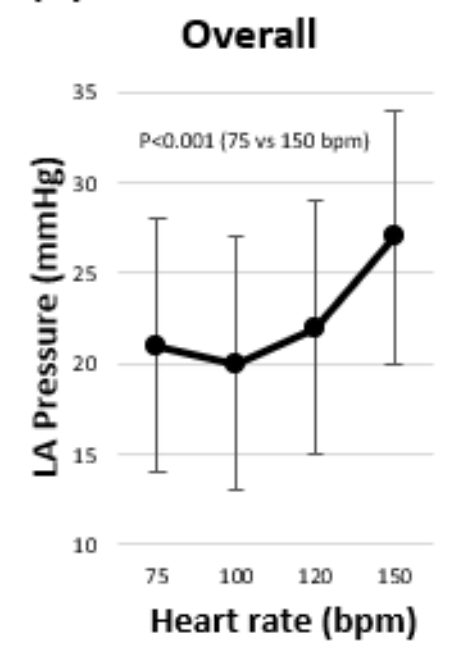

(B)

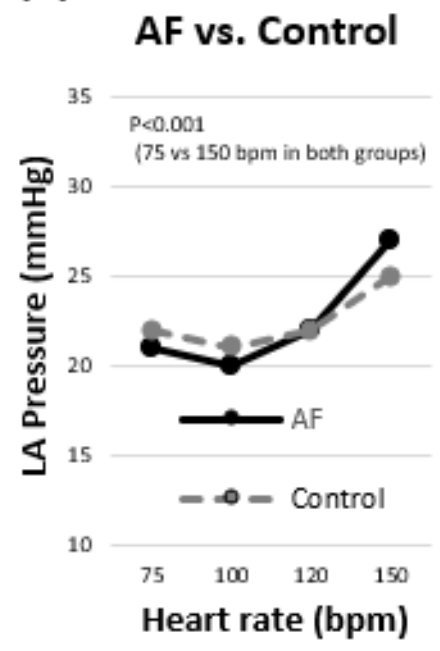

(c)

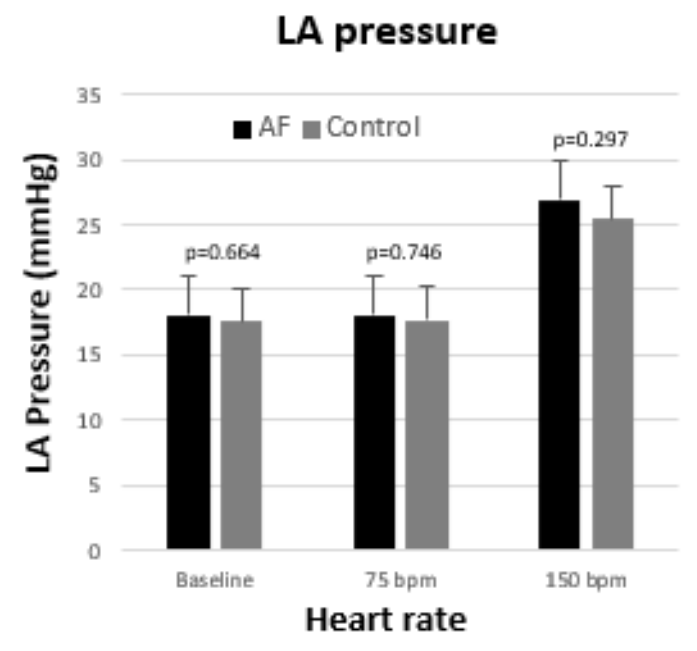

Figure 2. The pattern of heart rate dependent LA pressure response; (A) LA pressure was increased as heart rate change induced by right atrial pacing; $(B)$ In $A F$ and control groups, LA pressure response as heart rate change showed similar pattern; (C) The difference of $L A$ pressure in each heart rate between both groups. AF, atrial fibrillation; $L A$, left atrial. 
(A)

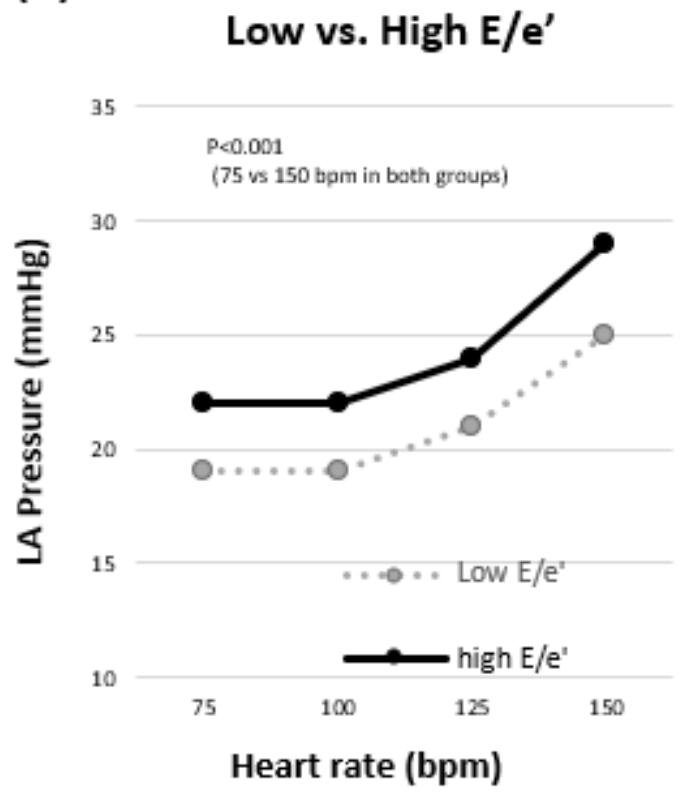

(B)

\section{LA pressure}

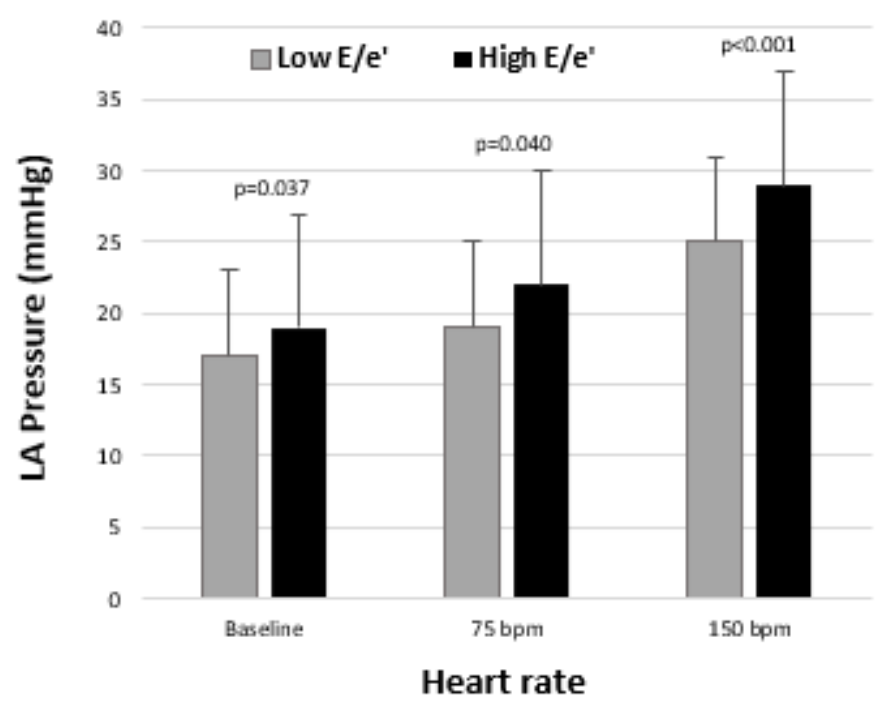

Figure 3. The different pattern of heart rate dependent LA pressure response; $(A)$ The different $L A$ pressure response between both low and high E/e' group; (B) In high E/e' groups, LA pressure response was significantly increased than that in low E/e' group. LA, left atrial.

(A)

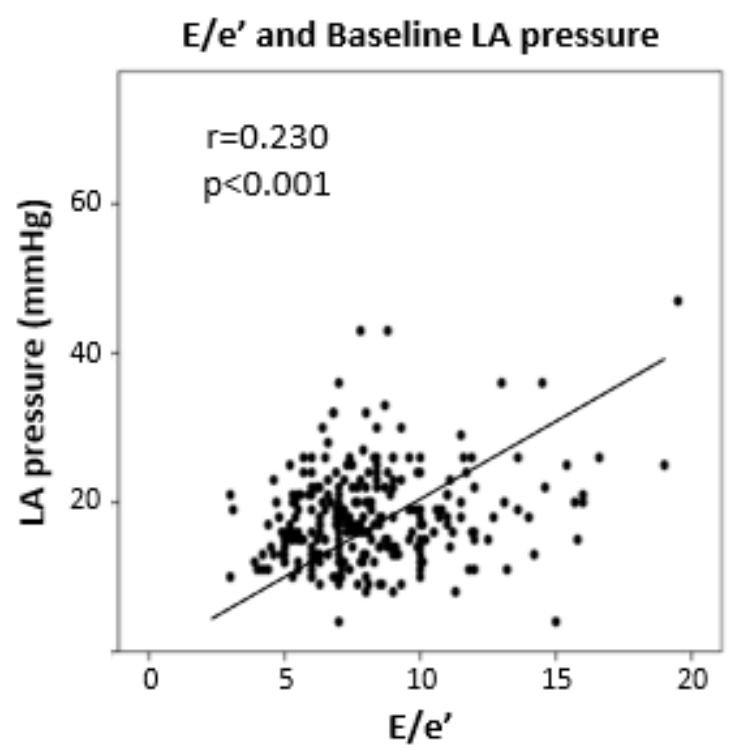

(B)

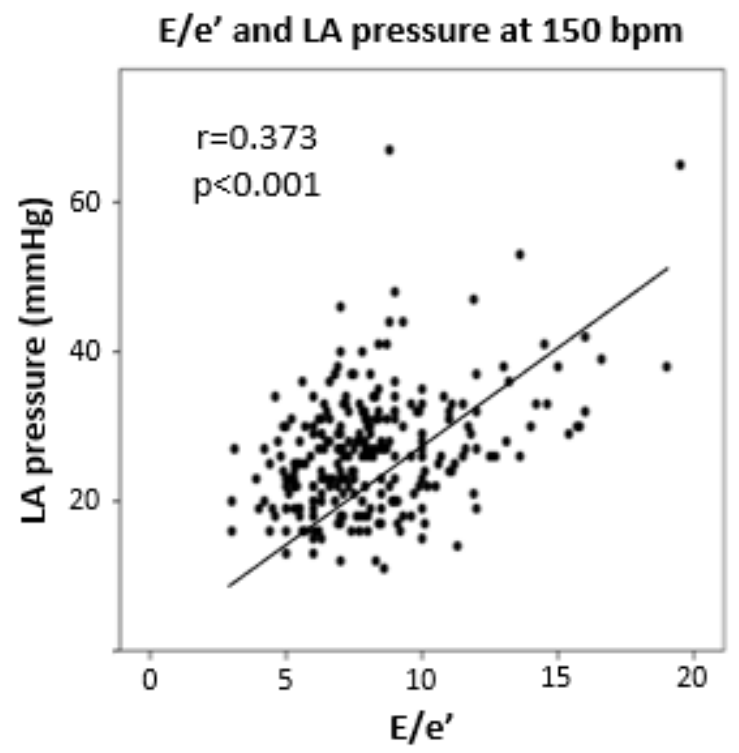

Figure 4. Correlation between left atrial pressure and Ele'; (A) at baseline; (B) at heart rate of $150 \mathrm{bpm}$; LA, left atrial. 
The presence of RAP-induced high LAPR could not predict AF recurrence after catheter ablation ( $\log$ rank $\mathrm{p}=0.299$ ) (Figure 5A). AF/AT freedom outcome was not different between the high and low LAPR groups (log rank $p=0.299$ ) (Figure 5B). It also did not differ between the high and low $\mathrm{E} / \mathrm{e}^{\prime}$ groups (log rank $\left.\mathrm{p}=0.541\right)$.

(A)

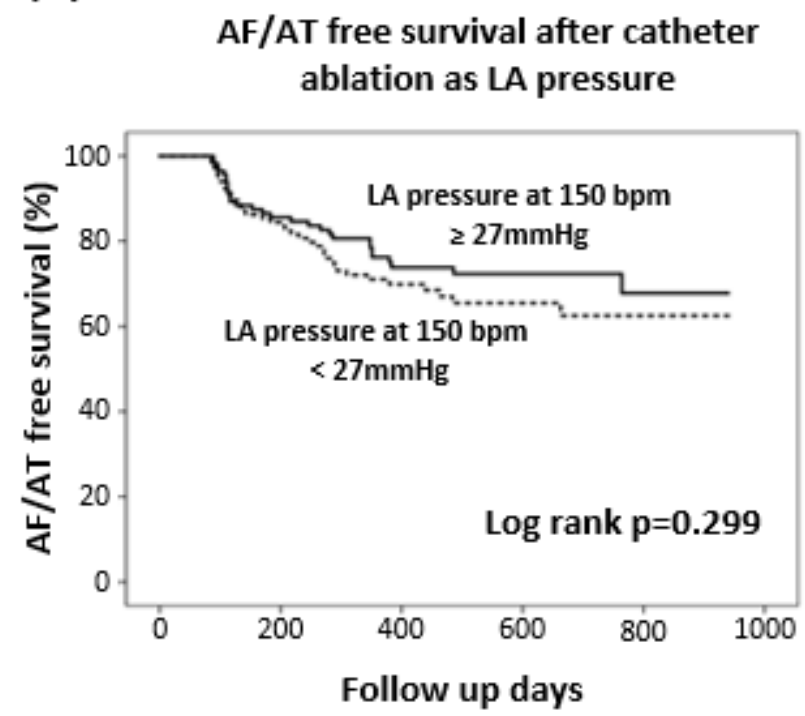

(B)

\section{AF/AT free survival after catheter ablation as E/e'}

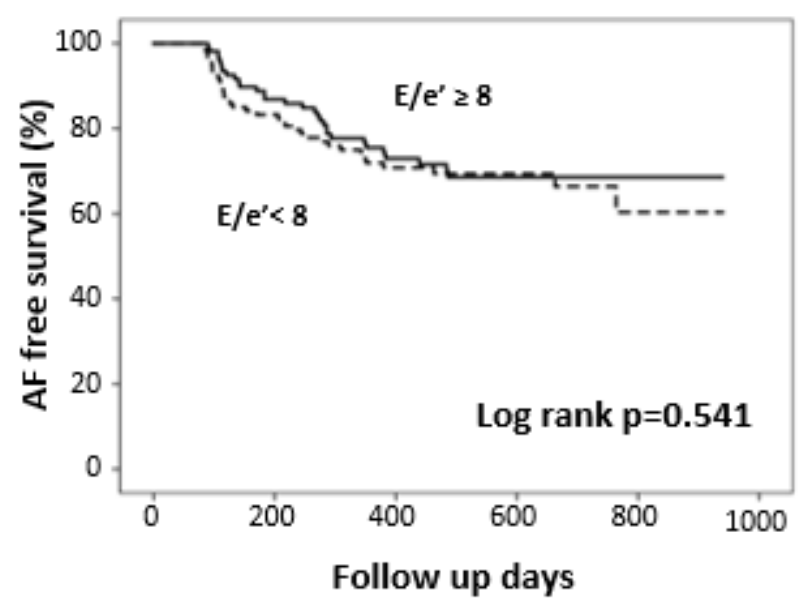

Figure 5. Atrial fibrillation/atrial tachycardia free survival as clinical outcome after catheter ablation. (A) High $(\geq 27 \mathrm{mmHg}$ at $150 \mathrm{bpm}$ ) versus low ( $<27 \mathrm{mmHg}$ at $150 \mathrm{bpm}$ ) left atrial pressure response group (B) High (E/e' $\geq 8$ versus low (E/e'< $<$ ) E/e' group. AF, atrial fibrillation; AT, atrial tachycardia; $L A$, left atrial.

Table 2. Comparison of clinical characteristics between high E/e' and low E/e' groups.

\begin{tabular}{|c|c|c|c|}
\hline & High E/e' group & Low $\mathrm{E} / \mathrm{e}^{\prime}$ group & \\
\hline & & $\mathrm{n}=144$ & $p$-value \\
\hline Age, y (SD) & $58(11)$ & $50(12)$ & $<0.001$ \\
\hline Male (\%) & $83(67)$ & $126(88)$ & $<0.001$ \\
\hline Body mass index, $\mathrm{kg} / \mathrm{m}^{2}(\mathrm{SD})$ & $25(3)$ & $24(3)$ & 0.136 \\
\hline Atrial fibrillation (\%) & $110(89)$ & $124(86)$ & 0.385 \\
\hline Hypertension (\%) & $56(45)$ & $34(24)$ & $<0.001$ \\
\hline Diabetes mellitus (\%) & $16(13)$ & $5(4)$ & 0.005 \\
\hline Myocardial infarction (\%) & $4(3)$ & 0 & 0.045 \\
\hline Cerebrovascular accident (\%) & $9(7)$ & $4(3)$ & 0.078 \\
\hline History of heart failure (\%) & $4(3)$ & $1(1)$ & 0.142 \\
\hline
\end{tabular}


Chronic kidney disease (\%)

Thyroid disease (\%)

\section{Echocardiographic data}

LVID in diastole, $\mathrm{mm}$ (SD)

LVID in systole, $\mathrm{mm}$ (SD)

LV mass, g (SD)

LV mass index, $\mathrm{g} / \mathrm{m}^{2}$ (SD)

LV Ejection fraction, \% (SD)

LA AP diameter, mm (SD)

$\mathrm{E}(\mathrm{SD})$

$\mathrm{e}^{\prime}(\mathrm{SD})$

$\mathrm{E} / \mathrm{e}^{\prime}$ ratio $(\mathrm{SD})$

DT of E (SD)

Estimated PASP, mmHg (SD)
$2(2)$

2 (1)

0.631

$6(5)$

$3(2)$

0.182

47 (4)

47 (4)

0.332

29 (5)

29 (4)

0.816

160 (33)

155 (32)

0.209

91 (16)

85 (16)

0.001

59 (3)

59 (3)

0.683

41 (6)

38 (6)

$<0.001$

70 (14)

57 (13)

$<0.001$

7 (2)

$9(2)$

$<0.001$

$10(2)$

$6(1)$

$<0.001$

175 (46)

$173(34)$

0.589

$30(6)$

$28(6)$

0.002

$L V I D=$ left ventricular internal diameter; $L V=$ left ventricular; $L A=$ left atrial; $A P=$ anterorposterior; $D T=$ deceleration time; PASP = pulmonary artery systolic pressure.

Table 3. Univariate and multivariate analysis (Logistic regression) showing odds ratio to predict high LA pressure response (LA pressure $>26 m m H g$ )

\begin{tabular}{|c|c|c|c|c|}
\hline \multirow{3}{*}{ Variables } & Univariate & \multicolumn{3}{|c|}{ Multivariate } \\
\hline & & $p$-Value & & $p$-Value \\
\hline & odds ratio $(95 \% \mathrm{CI})$ & & odds ratio $(95 \% \mathrm{CI})$ & \\
\hline Age, 1 year & $1.013(0.993-1.033)$ & 0.198 & & \\
\hline Female sex & $1.085(0.609-1.934)$ & 0.782 & & \\
\hline HTN & $1.279(0.769-2.126)$ & 0.343 & & \\
\hline Diabetes mellitus & $0.610(0.244-1.525)$ & 0.291 & & \\
\hline $\mathrm{AF}$ & $0.904(0.440-1.859)$ & 0.784 & & \\
\hline Body mass index, $\mathrm{kg} / \mathrm{m}^{2}$ & $1.111(1.021-1.208)$ & 0.014 & $1.098(1.006-1.197)$ & 0.035 \\
\hline
\end{tabular}


LA diameter, $\mathrm{mm}$

LV mass index

LV ejection fraction, \%

High E/e' (> 7.8)
$1.010(0.973-1.049)$

$1.018(1.003-1.034)$

$1.069(0.975-1.173)$

0.153

$2.336(1.430-3.818)$
$1.014(0.998-1.197)$

0.081

0.605

0.017

AF; Atrial fibrillation, LV; Left ventricular, LA; Left atrial, NT Pro BNP; N terminal brain natriuretic peptides

\section{Discussion}

LA pressure increased with incremental atrial pacing. The LAPR was not correlated with the presence of $\mathrm{AF}$, but was closely related to $\mathrm{E} / \mathrm{e}^{\prime}$, an echocardiographic marker of LV diastolic dysfunction.

\subsection{The mechanism of left atrial hypertension}

LA pressure is influenced by several factors such as LV systolic and diastolic function, LA chamber stiffness, and intravascular volume status. If LV diastolic function worsens, LV end-diastolic pressure (LVEDP) increases to maintain adequate LV stroke volume. [7] The LA is directly exposed to the LV pressure during its diastolic phase. The filling of the LA during the LV systolic phase produces high LA pressure, which leads to increased LA wall tension and remodeling. LA pressure reflects both LA remodeling in chronically increased LVEDP exposure and pressure loading through the mitral valve. It can be used to investigate the prognosis of HF. Increased LA pressure causes electrical heterogenicity of the atrial myocardium, which causes AF. [8,9] AF is a result as well as an aggravating factor of HF. We hypothesized that LA pressure and LAPR were increased in patients with AF, but there was no significant difference in the results. First, the degree of diastolic dysfunction was not significantly different between the AF and control groups. E/e' was statistically different, but the absolute values were not clearly different. Many subjects with relatively mild HF were included because patients with AF are candidates for ablation. Second, the dilated and remodeled atria compensate for the pressure change.

\subsection{The clinical implication of left atrial pressure response}

LA pressure does not increase at the normal range of heart rate but increases rapidly beyond its threshold. This means that insufficient time to fill the appropriate volume leads to an increase in LVEDP. In our study, LAPR was closely related to E/e' regardless of the rhythm status. These results imply that the main factor of LAPR is LV diastolic dysfunction rather than atrial remodeling. LV diastolic function determines the boundary value of the LAPR. Particularly, it is helpful in identifying the cause in patients who complain of non-ischemic exercise-related dyspnea. Increased LA pressure during exercise or tachycardia causes dyspnea, and E/e' can be a marker of exertional dyspnea of 
cardiac origin. At rest, patients with diastolic dysfunction may have a cardiac output or filling pressure similar to that of healthy individuals who have normal diastolic function. Exercise echocardiography [10] is usually performed to detect reduced LV systolic and/or diastolic reserve capacity in the setting of coronary disease or diastolic dysfunction. The result of exercise echocardiography can be predicted using E/e', which closely reflects LAPR.

\subsection{The clinical implication of E/e' as a marker of early left ventricular diastolic dysfunction}

E/e' measured by echocardiography is a non-invasive method that reflects LV diastolic dysfunction. LAPR is a marker of LV diastolic reservoir, but it has to be obtained using an invasive method. E/e' measured by echocardiography is a non-invasive method and was found to be closely correlated with the LAPR. [11] Several other studies have shown a good correlation between E/e and pulmonary capillary pressure or LV mean diastolic pressure during variable levels of exercise. $[12,13] \mathrm{E} / \mathrm{e}^{\prime}$ is clinically useful regardless of rhythm status.

Baseline LA pressure, LAPR, and E/e' were not able to predict the outcome after catheter ablation. The most relevant predictor of prognosis after catheter ablation is LA remodeling, including enlargement and fibrosis. The main determinant of both LA pressure and LAPR is LV diastolic dysfunction rather than LA remodeling. This study included only people with relatively mild HF. In other studies, increased E/e' was a predictor of poor outcome after ablation, such as low LA voltage. [14] As a result, it is impossible to determine a candidate for ablation considering E/e' in the early stages of HF. However, it can be helpful in deciding pre- and post-procedural medication, and what causes mainly provoke dyspnea.

\subsection{Study limitations}

The following limitations should be considered when interpreting this result. This was a single-center observational study that included only patients selected for catheter ablation of AF or supraventricular tachycardia. Therefore, it is difficult to generalize this finding to the entire population. Since most of the patients in the AF group had compensated for HF and proper general condition, there may not be any difference from the control group. This tends to ignore the effect of atrial remodeling on LA pressure. Next, the difference in rhythm status should be considered when LA pressure was measured. In patients with persistent $\mathrm{AF}$, LA pressure was measured after returning to SR after cardioversion; however, it may not have recovered from stunning even after $5 \mathrm{~min}$. However, this result was consistently observed in other patients who were measured without cardioversion. Lastly, LA pressure measured during tachycardia induced by pacing may differ from that during increased heart rate due to exercise and emotional changes in daily life. This is because the increase in heart rate by activity is accompanied by an increase in LV contractility, aortic stiffness, and preload in response to an increase in sympathetic tone. For this reason, it is difficult to mention that the results of this study perfectly reflect the heart response during ordinary exercise.

\section{Conclusions}

In conclusion, LA pressure showed a constant increase with the heart rate change according to pacing. The echocardiographic non-invasive marker, E/e', reflected the LAPR 
measured during incremental pacing. It can be an indication to evaluate the cause of exertional dyspnea regardless of AF.

Author Contributions: Conceptualization, S-Y.R., K-N.L. and Y-H.K.; data curation, M.I., A.J. and S.T.; formal analysis, S-Y.R.; investigation, M.I., A.J. and S.T.; methodology, S-Y.R., K-N.L. and Y-S.B.; validation, J.S.; writing-original draft, S-Y.R. and K-N.L.; writing-review and editing, J-I.C. and Y-H.K. All authors have read and agreed to the published version of the manuscript.

Funding: This research received no external funding for this study.

Institutional Review Board Statement: The studies were conducted according to the principles of the Declaration of Helsinki and were approved by the Institutional Review Board of the Korea University Anam Hospital (2016AN0152). The participants provided informed written consent prior to participating in these studies.

Informed Consent Statement: Informed consent was obtained from all subjects involved in the study.

Data Availability Statement: The data presented in this study are available on request from the corresponding author. The data are not publicly available due to privacy restrictions.

Conflicts of Interest: The authors declare no conflict of interest.

\section{References}

1. Senni, M.; Tribouilloy, C.M.; Rodeheffer, R.J.; Jacobsen, S.J.; Evans, J.M.; Bailey, K.R.; Redfield, M.M. Congestive heart failure in the community: A study of all incident cases in olmsted county, minnesota, in 1991. Circulation 1998, 98, 2282-2289.

2. Vasan, R.S.; Larson, M.G.; Benjamin, E.J.; Evans, J.C.; Reiss, C.K.; Levy, D. Congestive heart failure in subjects with normal versus reduced left ventricular ejection fraction: Prevalence and mortality in a population-based cohort. J Am Coll Cardiol 1999, 33, 1948-1955.

3. Obokata, M.; Olson, T.P.; Reddy, Y.N.V.; Melenovsky, V.; Kane, G.C.; Borlaug, B.A. Haemodynamics, dyspnoea, and pulmonary reserve in heart failure with preserved ejection fraction. Eur Heart J 2018, 39, 2810-2821.

4. Park, J.; Joung, B.; Uhm, J.S.; Young Shim, C.; Hwang, C.; Hyoung Lee, M.; Pak, H.N. High left atrial pressures are associated with advanced electroanatomical remodeling of left atrium and independent predictors for clinical recurrence of atrial fibrillation after catheter ablation. Heart Rhythm 2014, 11, 953-960.

5. Kalifa, J.; Jalife, J.; Zaitsev, A.V.; Bagwe, S.; Warren, M.; Moreno, J.; Berenfeld, O.; Nattel, S. Intra-atrial pressure increases rate and organization of waves emanating from the superior pulmonary veins during atrial fibrillation. Circulation 2003, 108, 668-671.

6. Markowitz, S.M. Left atrial hypertension in atrial fibrillation: Dealing with the pressure. JACC Clin Electrophysiol 2017, 3, 470-472.

7. Thomas, L.; Marwick, T.H.; Popescu, B.A.; Donal, E.; Badano, L.P. Left atrial structure and function, and left ventricular diastolic dysfunction: Jacc state-of-the-art review. J Am Coll Cardiol 2019, 73, 1961-1977.

8. Tsang, T.S.; Gersh, B.J.; Appleton, C.P.; Tajik, A.J.; Barnes, M.E.; Bailey, K.R.; Oh, J.K.; Leibson, C.; Montgomery, S.C.; Seward, J.B. Left ventricular diastolic dysfunction as a predictor of the first diagnosed nonvalvular atrial fibrillation in 840 elderly men and women. J Am Coll Cardiol 2002, 40, 1636-1644.

9. Vasan, R.S.; Larson, M.G.; Levy, D.; Galderisi, M.; Wolf, P.A.; Benjamin, E.J.; National Heart, L.; Blood Institute, N.I.o.H. Doppler transmitral flow indexes and risk of atrial fibrillation (the framingham heart study). Am J 
Cardiol 2003, 91, 1079-1083.

10. Ha, J.W.; Oh, J.K.; Pellikka, P.A.; Ommen, S.R.; Stussy, V.L.; Bailey, K.R.; Seward, J.B.; Tajik, A.J. Diastolic stress echocardiography: A novel noninvasive diagnostic test for diastolic dysfunction using supine bicycle exercise doppler echocardiography. J Am Soc Echocardiogr 2005, 18, 63-68.

11. Hummel, Y.M.; Liu, L.C.Y.; Lam, C.S.P.; Fonseca-Munoz, D.F.; Damman, K.; Rienstra, M.; van der Meer, P.; Rosenkranz, S.; van Veldhuisen, D.J.; Voors, A.A. et al. Echocardiographic estimation of left ventricular and pulmonary pressures in patients with heart failure and preserved ejection fraction: A study utilizing simultaneous echocardiography and invasive measurements. Eur J Heart Fail 2017, 19, 1651-1660.

12. Burgess, M.I.; Jenkins, C.; Sharman, J.E.; Marwick, T.H. Diastolic stress echocardiography: Hemodynamic validation and clinical significance of estimation of ventricular filling pressure with exercise. J Am Coll Cardiol 2006, 47, 1891-1900.

13. Ritzema, J.L.; Richards, A.M.; Crozier, I.G.; Frampton, C.F.; Melton, I.C.; Doughty, R.N.; Stewart, J.T.; Eigler, N.; Whiting, J.; Abraham, W.T. et al. Serial doppler echocardiography and tissue doppler imaging in the detection of elevated directly measured left atrial pressure in ambulant subjects with chronic heart failure. JACC Cardiovasc Imaging 2011, 4, 927-934.

14. Masuda, M.; Fujita, M.; Iida, O.; Okamoto, S.; Ishihara, T.; Nanto, K.; Kanda, T.; Sunaga, A.; Tsujimura, T.; Matsuda, Y. et al. An e/e' ratio on echocardiography predicts the existence of left atrial low-voltage areas and poor outcomes after catheter ablation for atrial fibrillation. Europace 2018, 20, e60-e68. 